\title{
TRAIL limits excessive host immune responses in bacterial meningitis
}

\author{
Olaf Hoffmann, ${ }^{1}$ Josef Priller, ${ }^{1,2}$ Timour Prozorovski, ${ }^{3}$ Ulf Schulze-Topphoff, ${ }^{3}$ \\ Nevena Baeva, ${ }^{2}$ Jan D. Lunemann, ${ }^{3}$ Orhan Aktas, ${ }^{3}$ Cordula Mahrhofer, ${ }^{1}$ \\ Sarah Stricker,,$^{1,2}$ Frauke Zipp, ${ }^{3}$ and Joerg R. Weber ${ }^{1,4}$
}

${ }^{1}$ Department of Neurology, ${ }^{2}$ Laboratory of Molecular Psychiatry, ${ }^{3}$ Department of Neuroimmunology, and ${ }^{4}$ Department of Cell Biology and Neurobiology, Charité — Universitätsmedizin Berlin, Berlin, Germany.

\begin{abstract}
Apart from potential roles in anti-tumor surveillance, the TNF-related apoptosis-inducing ligand (TRAIL) has important regulatory functions in the host immune response. We studied antiinflammatory effects of endogenous and recombinant TRAIL (rTRAIL) in experimental meningitis. Following intrathecal application of pneumococcal cell wall, a TLR2 ligand, we found prolonged inflammation, augmented clinical impairment, and increased apoptosis in the hippocampus of TRAIL $-/-$ mice. Administration of rTRAIL into the subarachnoid space of $\mathrm{TRAIL}^{-/-}$mice or reconstitution of hematopoiesis with wild-type bone marrow cells reversed these effects, suggesting an autoregulatory role of TRAIL within the infiltrating leukocyte population. Importantly, intrathecal application of rTRAIL in wild-type mice with meningitis also decreased inflammation and apoptosis. Moreover, patients suffering from bacterial meningitis showed increased intrathecal synthesis of TRAIL. Our findings provide what we believe is the first evidence that TRAIL may act as a negative regulator of acute CNS inflammation. The ability of TRAIL to modify inflammatory responses and to reduce neuronal cell death in meningitis suggests that it may be used as a novel antiinflammatory agent in invasive infections.
\end{abstract}

\section{Introduction}

Previous research on TNF-related apoptosis-inducing ligand (TRAIL; also termed APO2L) has mostly focused on its ability to induce apoptosis in transformed cells but not in most normal cells. TRAIL is a potential antitumor therapy (1). In addition, there is a growing understanding of the physiological functions of endogenous TRAIL in immunity. TRAIL death receptors and decoy receptors are present on peripheral blood leukocytes (2-6) and may play an important role in antitumor surveillance by the immune system (7) and in the regulation of cell survival.

Inhibitory effects of TRAIL on (auto)antigen-specific T lymphocytes or thymocytes were demonstrated not only in vitro (8) but also in vivo in models such as experimental autoimmune encephalomyelitis (9) and autoimmune arthritis $(10,11)$. Accordingly, soluble TRAIL (sTRAIL) levels were upregulated in multiple sclerosis patients treated with immune-regulatory interferon $\beta$ therapy and highest in patients responding to this therapy (12). Recently, the TRAIL system has been implicated in the elimination of senescent circulating neutrophils $(4,13)$ as well as in the clearance of activated tissue neutrophils (14).

Leukocytes are the first line of defense in acute bacterial infections but have also been identified as key effectors of damage in bacterial meningitis (15-17). Acute bacterial meningitis is still a life-threatening invasive infectious disease. Despite effective antibiotic treatment, mortality remains as high as $30 \%$, and only half of the survivors return to work $(18,19)$. Clinical complications, such as the formation of brain edema, increased intracranial pres-

Nonstandard abbreviations used: AOEB, acridine orange plus ethidium bromide; CSF, cerebrospinal fluid; CXCL12, CXC chemokine ligand 12; MPO, myeloperoxidase; PCW, pneumococcal cell wall; rTRAIL, recombinant TRAIL; sTRAIL, soluble TRAIL; TRAIL, TNF-related apoptosis-inducing ligand; TRAIL-R2, TRAIL receptor 2. Conflict of interest: The authors have declared that no conflict of interest exists. Citation for this article: J. Clin. Invest. 117:2004-2013 (2007). doi:10.1172/JCI30356. sure, and alterations of the cerebral blood flow, contribute to the unfavorable outcome. These alterations are largely mediated by host immune responses and leukocyte function $(15,20,21)$. Additionally, there is a substantial risk of long-term neuropsychological sequelae in survivors $(22,23)$. Such impairment has been linked to neuronal loss, mostly by apoptosis, which is particularly pronounced in the hippocampus (24-27). Experimental data in meningitis caused by Streptococcus pneumoniae - the most frequent and disastrous pathogen in meningitis - suggest that bacterial toxins, such as the pore-forming pneumolysin or hydrogen peroxide, mediate approximately $50 \%$ of the neuronal cell loss while the remaining neuronal damage is driven by host immune responses (25-27). In the context of antibiotic therapy, it is highly desirable to limit the inflammatory process in order to prevent such acute and chronic complications (28).

Today, evidence-based adjunctive treatment of meningitis is limited to dexamethasone $(19,29)$. In pneumococcal meningitis in particular, mortality and frequency of complications are reduced by this treatment (29), but important issues surround the question of whether glucocorticoids impair the penetration of antibiotics into the cerebrospinal fluid (CSF) $(30,31)$ or worsen the neuronal damage (32). Specifically, neutrophil survival is prolonged by glucocorticoids $(33,34)$; this raises the risk of cytotoxic effects.

In the present study, we found elevated levels of sTRAIL in the CSF of patients with bacterial meningitis. We hypothesized that the TRAIL system is an essential regulator of leukocyte survival in the CSF during meningitis and that recombinant TRAIL (rTRAIL) could be used to modulate the inflammatory response in invasive infections, such as bacterial meningitis.

\section{Results}

sTRAIL is elevated in the CSF of patients with bacterial meningitis. The concentration of STRAIL in the CSF (Figure 1A) and the CSF to serum ratio (Figure 1B) were significantly increased in patients 
A

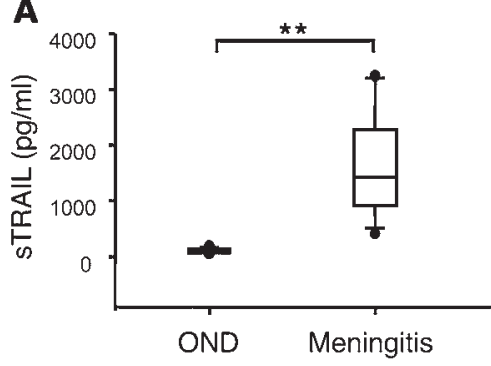

B

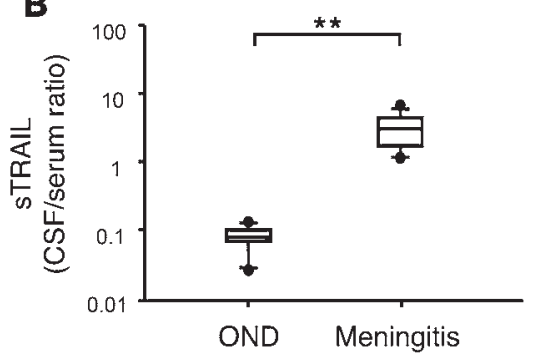

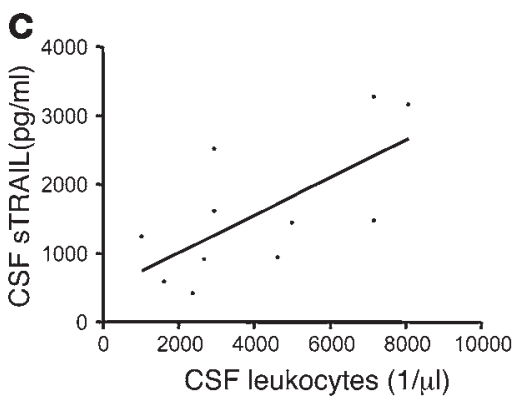

Figure 1

TRAIL is released into the CSF of patients with acute meningitis. (A) In CSF samples from patients with acute bacterial meningitis, the concentration of sTRAIL is significantly higher than in patients with other noninflammatory diseases (OND). ${ }^{* *} P<0.01$, Student's $t$ test. (B) The increased CSF/serum ratio supports an intrathecal source of STRAIL during meningitis. ${ }^{* *} P<0.01$, Student's $t$ test. (C) The concentration of TRAIL in CSF correlated significantly with the cell count. Spearman's $r=0.717 ; P=0.016$.

diagnosed with bacterial meningitis compared with control patients. The level of sTRAIL correlated significantly with the leukocyte count in the CSF (Figure 1C). These data indicate that sTRAIL is released into the CSF compartment, suggesting a specific role for TRAIL in bacterial meningitis.

Effect of TRAIL genotype on the influx of leukocytes in experimental meningitis. Intrathecal application of pneumococcal cell wall (PCW), an established model of experimental meningitis in mice (35), caused a pronounced influx of leukocytes into the CSF ( $n=5$ to 10 animals/genotype and time point). In wild-type mice, pleocytosis was highest at 12 hours and resolved almost completely within 24 hours after meningitis induction (Figure 2A). In TRAIL - $^{-}$ mice, we observed a delayed and protracted influx of leukocytes, with the highest concentration present at 18 hours and persistence at 48 hours. While the TRAIL genotype had no significant impact on the differential hemograms of untreated mice (Supplemental Figure 1A; supplemental material available online with this article; doi:10.1172/JCI30356DS1), we observed a substantial effect on the kinetics of leukocyte subsets in the CSF after meningitis induction (Figure 2B): in wild-type mice, an initial granulocytic phase was followed by rapid clearance of granulocytes and a predominance of mononuclear cells. In comparison, granulocytes were much less abundant in the CSF of TRAIL ${ }^{-/-}$mice during early meningitis, and their percentage did not decrease during the experimental period. At 12 hours, the difference in the absolute number of granulocytes (on average, $10,450 / \mu \mathrm{l}$ in wild-type mice versus $1,640 / \mu \mathrm{l}$ in TRAIL ${ }^{-/-}$mice; difference: $8,810 / \mu \mathrm{l}$ ) determined the difference in the total number of CSF leukocytes (on average, 12,440 leukocytes/ $\mu$ in wild-type mice versus 5,656 leukocytes/ $\mu \mathrm{l}$ in TRAIL - $^{--}$mice; difference: $6,784 / \mu l)$. These data suggest important effects of TRAIL on CSF leukocyte kinetics in meningitis.

We performed immunostainings of brain sections to determine whether the degree of tissue infiltration by granulocytes was also modulated by the TRAIL genotype. In general, few myeloperoxidase-

\section{Figure 2}

immunoreactive (MPO-immunoreactive) cells were identified in the brain, usually with highest abundance in the subcortical white matter. At 12 hours after challenge, $5.7 \pm 1.0 \mathrm{MPO}$-positive cells $/ \mathrm{mm}^{2}$ were found in the brain tissue of wild-type mice compared with $1.9 \pm 1.7$ cells $/ \mathrm{mm}^{2}$ in TRAIL ${ }^{-1-}$ mice $(P<0.05)$. At 18
A
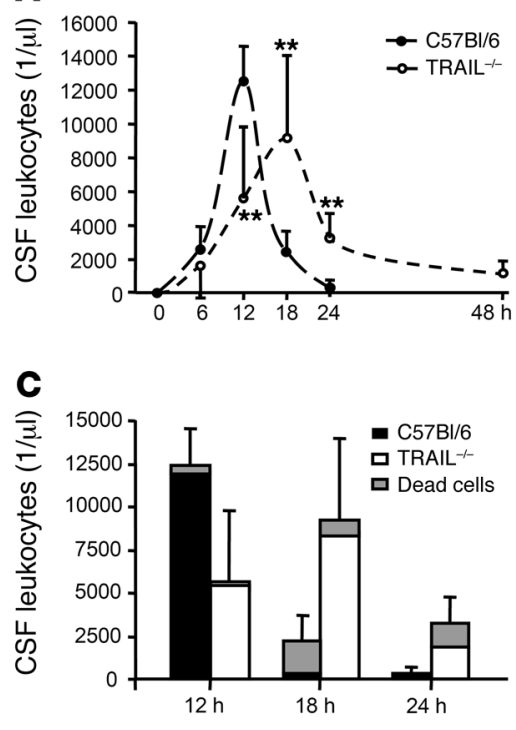

B

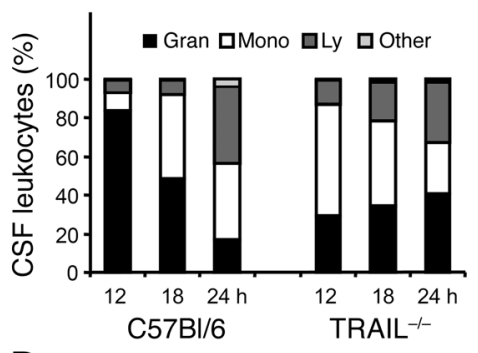

D

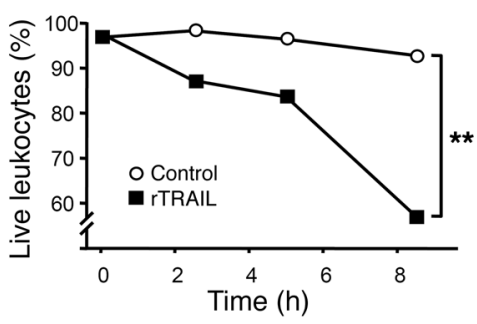

Leukocyte viability and persistence in the CSF are increased in TRAIL-- mice. (A) CSF leukocyte counts at specified time points following meningitis induction. Compared with wild-type C57BL/6 mice, TRAIL ${ }^{-1}$ mice show delayed influx of leukocytes into the CSF and delayed resolution of inflammation. ${ }^{* *} P<0.01$, Student's $t$ test. (B) Relative proportions of leukocyte subpopulations in the CSF of wild-type and TRAIL ${ }^{-1-}$ mice at 12,18 , and 24 hours after meningitis induction. Granulocytes (Gran) predominate in the early phase of experimental meningitis in wild-type mice while their accumulation is delayed in TRAIL-/- mice. Mono, monocytes; Ly, lymphocytes. (C) Proportion of nonviable cells of the total CSF leukocyte concentration at 12, 18, and 24 hours after meningitis induction in wild-type mice and TRAIL ${ }^{-/-}$mice as identified by AOEB staining (see also Supplemental Figure 1). Prolonged survival of activated CSF leukocytes is observed in TRAIL ${ }^{-/}$mice. $P<0.01$ at 18 hours and at 24 hours; Student's $t$ test. (D) Leukocyte apoptosis in pooled CSF samples from TRAIL ${ }^{-/-}$mice at 12 hours after meningitis induction. One representative experiment out of 2 is shown. rTRAIL $(100 \mathrm{ng} / \mathrm{ml})$ accelerates leukocyte apoptosis ex vivo. ${ }^{*} P<0.01$, log rank test. 
Table 1

Expression of cytokine mRNA levels

\begin{tabular}{|c|c|c|c|c|c|c|}
\hline & $\operatorname{Tnfa}\left(10^{-6}\right)$ & Ifng $\left(10^{-8}\right)$ & Cxc/12 (10-5) & $\operatorname{Tgfb}\left(10^{-5}\right)$ & I/4 (10-7) & $/ 110\left(10^{-8}\right)$ \\
\hline C57BL/6 & $1.13 \pm 1.24$ & $2.37 \pm 3.74$ & $5.84 \pm 5.46$ & $1.61 \pm 1.49$ & ND & $1.81 \pm 4.06$ \\
\hline TRAIL ${ }^{-/}$ & $6.20 \pm 3.53^{\mathrm{A}}$ & $5.23 \pm 9.39$ & $15.40 \pm 5.52$ & $5.44 \pm 2.40^{\mathrm{A}}$ & $1.16 \pm 2.37$ & $10.90 \pm 7.25$ \\
\hline
\end{tabular}

Shown is expression of cytokine mRNA levels in the cerebellum of wild-type C57BL/6 and TRAIL ${ }^{-1}$ mice at 18 hours to 24 hours after intrathecal challenge with PCW. Values are expressed relative to GAPDH mRNA (means $\pm \mathrm{SD}$ ). ND, not detected. ${ }^{A} P<0.05$, Student's $t$ test. of pro- to antiinflammatory cytokine mRNAs in wild-type mice versus $\mathrm{TRAIL}^{-/-}$mice.

Effect of TRAIL genotype on apoptosis in the dentate gyrus. Twentyfour hours after intrathecal application of PBS, the baseline rate of TUNEL-positive cells in the dentate gyrus was slightly lower in wild-type mice than in TRAIL $^{-/-}$mice ( $n=4$ /group). hours, $1.0 \pm 0.5 \mathrm{MPO}$-positive cells $/ \mathrm{mm}^{2}$ were present in wild-type mice compared with $1.5 \pm 1.0$ cells $/ \mathrm{mm}^{2}$ in TRAIL $^{-/-}$mice $(P=0.44)$. MPO-immunoreactive cells were extremely rare within the dentate gyrus ( 1 cell in 1 out of 21 animals). Moreover, double staining for MPO with the TUNEL reaction did not identify apoptotic brain cells in the vicinity of MPO-positive leukocytes. Cells stained for both TUNEL and MPO were present in the subarachnoid space but not within the brain parenchyma (data not shown).

Effect of TRAIL on leukocyte viability in the CSF. CSF samples were examined for the percentage of viable leukocytes using acridine orange plus ethidium bromide (AOEB) staining (Figure 2C and Supplemental Figure $1 ; n=3$ to 6 animals/group). At 12 hours after meningitis induction, AOEB staining demonstrated viability of virtually all leukocytes regardless of the TRAIL genotype. In wild-type mice, the vast majority of the leukocytes still present at 18 hours and 24 hours were dead. In contrast, TRAIL $/ /-$ mice had significantly more leukocytes in the CSF, and most of these cells remained viable even at 24 hours after challenge. All findings were supported by TUNEL staining (Supplemental Figure 1) and suggest a longer survival of invading leukocytes in TRAIL-/- mice during meningitis. In pooled CSF collected from $\mathrm{TRAIL}^{-/}$mice 12 hours after meningitis induction ( 2 independent studies, $n=3$ and $n=6$ ), addition of $100 \mathrm{ng} / \mathrm{ml}$ rTRAIL accelerated leukocyte apoptosis ex vivo as indicated by AOEB staining (log rank test, $P<0.01$; Figure 2D).

Effect of TRAIL genotype on cytokine $m R N A$ expression levels during meningitis. We also performed tests to determine whether TRAIL modulated the cytokine balance in the brain in meningitis. Measurements of cytokine levels in the CSF were impossible due to the small sample volumes. Real-time PCR was performed on cerebellar tissue homogenates and revealed higher mRNA expression levels of proinflammatory cytokines (Tnfa, Ifng, and CXC chemokine ligand 12 [Cxcl12]) and antiinflammatory cytokines $(T g f b, I l 4$, and Il10) in TRAIL Tl- $^{-1}$ mice compared with wild-type mice at 18 hours and 24 hours after intrathecal challenge with PCW $(P<0.01$ for Tnfa and Tgfb; Table 1$)$. No difference was observed for the ratio

Figure 3
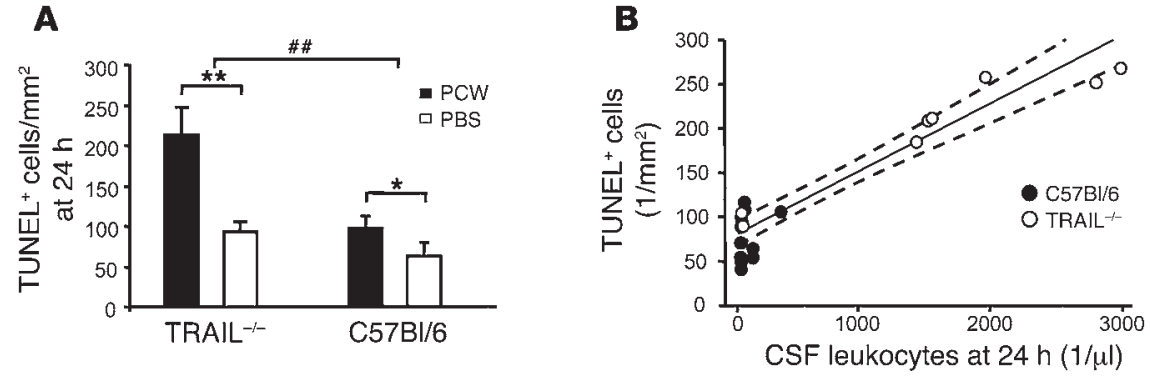

C

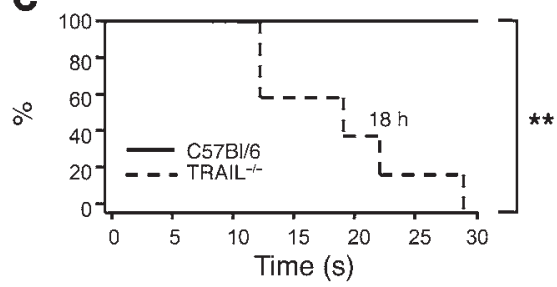

D

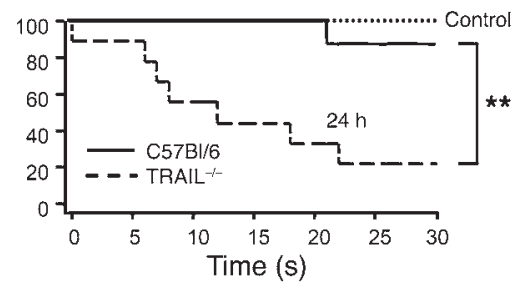

Apoptosis in the hippocampus and clinical impairment are augmented in TRAIL ${ }^{-1}$ mice with meningitis. (A) Twenty-four hours after meningitis induction, there is an increase in the number of TUNEL-positive apoptotic cells $/ \mathrm{mm}^{2}$ of the dentate gyrus in TRAlL ${ }^{-/}$mice and wild-type mice compared with that in PBS-treated controls. ${ }^{\star} P<0.05 ;{ }^{\star \star} P<0.01,1$-way ANOVA with Student-Neuman-Keuls post hoc analysis. Meningitis caused a much stronger increase of apoptotic cell death in TRAIL-- mice than in wild-type mice compared with controls. ${ }^{\# \#} P<0.01$, Student's $t$ test. Examples of TUNEL-stained cells are shown in Supplemental Figure 2. (B) Correlation of the number of TUNEL-positive cells $/ \mathrm{mm}^{2}$ in the dentate gyrus with the concentration of CSF leukocytes at 24 hours after meningitis induction. Multiple regression analysis identified the leukocyte count as a stronger predictor of damage than the genotype $\left(T_{\text {leukocytes }}=7.2 ; T_{\text {genotype }}=2.0\right)$. $(\mathbf{C}$ and $\mathbf{D})$ Motor and coordination assessment with the tightrope test at 18 hours (C) and 24 hours (D) after meningitis induction. Clinical impairment is more pronounced in TRAIL ${ }^{-1}$ mice compared with wild-type mice. ${ }^{* \star} P<0.01$, log rank test. All control mice were able to hold on to the rope for at least 30 seconds. 
A

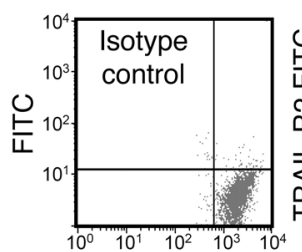

GR-1 PE
B

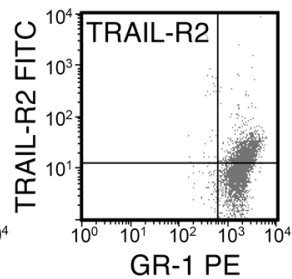

c

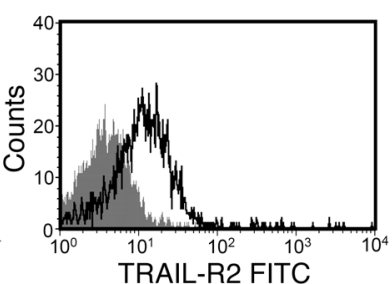

\section{D}

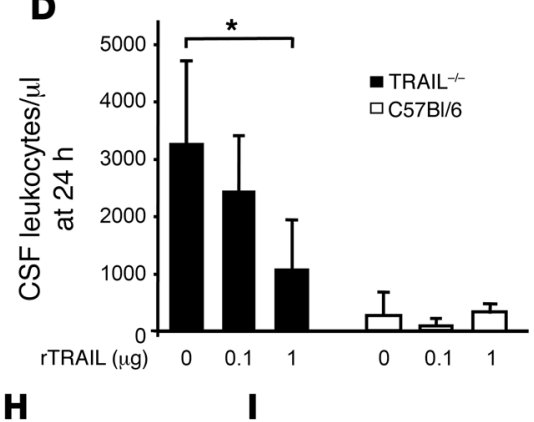

$\mathbf{F}$

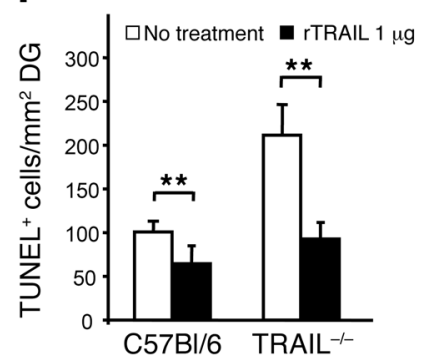

G

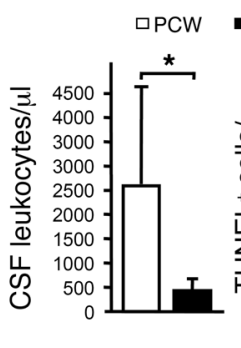

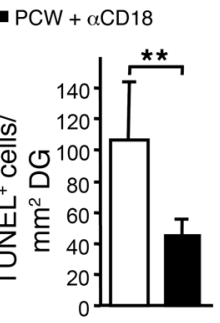

I

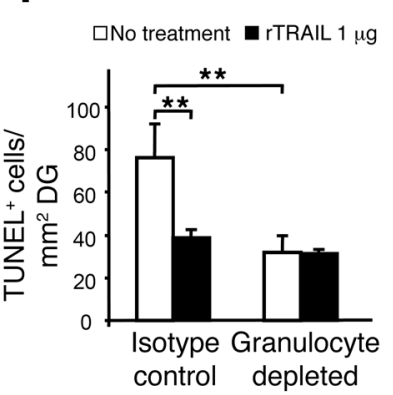

\section{Figure 4}

rTRAIL modulates inflammation and apoptosis in the hippocampus of wild-type and TRAIL ${ }^{-/-}$mice with meningitis. (A-C) FACS analysis of murine peripheral blood demonstrates the presence of TRAIL-R2 on Gr-1-positive leukocytes. (A) Gr-1 staining and isotype control staining for TRAIL-R2. PE, phycoerthrin. (B) Double staining for Gr-1 and TRAIL-R2. (C) Histogram plot of TRAIL-R2 expression in the gated Gr-1 high population. Isotype control (gray curve); TRAIL-R2 (black curve). (D-F) Effects of treatment with rTRAIL in experimental meningitis. (D) In TRAIL ${ }^{-1-}$ mice, a dose-dependent reduction of pleocytosis was observed 24 hours after intrathecal administration of $0.1-1 \mu \mathrm{g}$ rTRAIL and PCW. In wild-type mice, rTRAIL had no effect. (E) At 12 hours after meningitis induction, coapplication of $1 \mu \mathrm{g}$ rTRAIL with PCW reduced the levels of pleocytosis in wild-type mice and TRAIL ${ }^{--}$mice. (F) rTRAIL reduced apoptosis in the dentate gyrus at 24 hours after meningitis induction in wild-type and TRAIL ${ }^{-/}$mice. (G) Parenteral administration of an antibody against CD18 significantly reduced the recruitment of leukocytes across the blood-brain barrier in wild-type mice. (H) CD18 inhibition also diminished the number of TUNEL-positive cells in the dentate gyrus at 24 hours after meningitis induction in wild-type mice. (D-H) ${ }^{\star} P<0.05$; ${ }^{* \star} P<0.01$, Student's $t$ test or Mann-Whitney $U$ test as appropriate. (I) In TRAIL $^{--}$mice, depletion of granulocytes with an antibody against Ly6G reduced the number of TUNEL-positive cells in the dentate gyrus at 24 hours after meningitis induction. ${ }^{* *} P<0.01$, Student's $t$ test. Intrathecal treatment with $1 \mu \mathrm{g}$ rTRAlL diminished apoptosis in the dentate gyrus of nondepleted mice at 24 hours after meningitis induction $(P<0.01$, Student's $t$ test), but no additional effect of rTRAIL was present in granulocytedepleted mice. $P$ = NS, 2-way ANOVA. DG, dentate gyrus.

Expression of TRAIL receptor 2 on granulocytes. We assumed a regulatory role of TRAIL on the apoptosis of activated granulocytes in the CSF. Using FACS analysis, we demonstrated the presence of the TRAIL death receptor TRAIL receptor 2 (TRAIL-R2) on Gr-1-positive peripheral blood leukocytes in untreated wildtype mice (Figure 4, A-C). Additional staining was performed on brain sections from wild-type mice at 12 hours after meningitis induction. Here, TRAIL-R2 immunoreactivity was present mainly on microglia and on the majority of infiltrating granulocytes identified by their MPO expression (Supplemental Figure 3A).

Intrathecal administration of $r$ TRAIL restores leukocyte clearance in the CSF. Intrathecal administration of rTRAIL in PBS-challenged animals did not induce leukocyte influx into the CSF of wild-type mice $(n=3)$ or TRAIL ${ }^{-/-}$mice $(n=6)$ at 24 hours after surgery. In TRAIL ${ }^{-/}$animals with meningitis, we observed a dose-dependent reduction of the number of CSF leukocytes at 24 hours after meningitis induction (Figure 4D; $n=4$ /group). In wild-type mice with meningitis, the low CSF cell count at 24 hours was not significantly modulated by rTRAIL ( $n=4$ /group). Having determined that $1 \mu \mathrm{g}$ rTRAIL was required for the reduction of CSF leukocyte counts, we went on to explore the effects of rTRAIL administration at an earlier time point after meningitis induction. Administration of $1 \mu \mathrm{g}$ rTRAIL/animal resulted in a marked decrease in CSF leukocyte counts at 12 hours after meningitis induction (Figure 4E) in both TRAIL $^{-/-}$and wild-type mice ( $n=6 /$ group).

rTRAIL reduces apoptosis in the hippocampus after experimentalmeningitis. Next, we determined the effect of rTRAIL on the rate of apoptosis in the dentate gyrus at 24 hours after meningitis induction (Figure 4F). In $\mathrm{TRAIL}^{-/-}$mice with meningitis, intrathecal administration of $1 \mu \mathrm{g}$ rTRAIL led to a marked reduction of TUNEL-positive cells in the dentate gyrus. In fact, the levels of TUNEL-positive cells were similar to those of wild-type animals with meningitis. Moreover, administration of rTRAIL reduced the number of TUNEL-positive cells in the dentate gyrus even in wild-type mice with meningitis. rTRAIL had no effect on the number of TUNEL-positive cells in the dentate gyrus of PBS-challenged animals of either genotype (data not shown).

Effects of CD18 inhibition or granulocyte depletion in experimental meningitis. In wild-type mice, parenteral treatment with the blocking monoclonal anti-CD18 antibody GAME46 $(n=7)$ effectively inhibited leukocyte recruitment to the CSF (Figure 4G) and reduced the number of TUNEL-positive cells in the dentate gyrus 24 hours after meningitis induction compared with those in mice challenged with PCW alone $(n=8)$. Granulocytic depletion of TRAIL - $^{-1}$ mice 

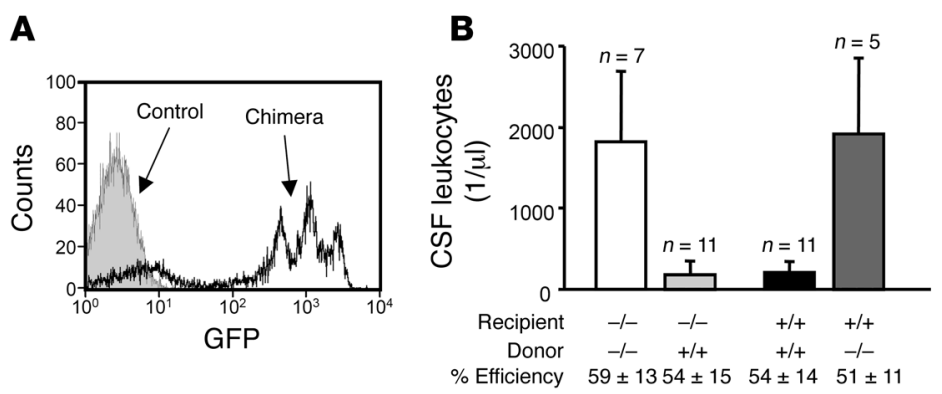

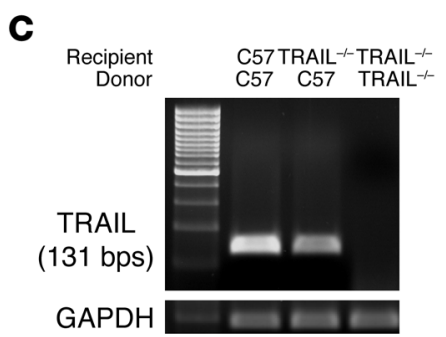

\section{Figure 5}

Myeloid cells are the source of TRAIL in experimental meningitis. (A) In BM chimeric mice, the degree of chimerism was assessed by FACS analysis of peripheral blood leukocytes. Histogram plot demonstrates strong enhanced GFP expression in leukocyte populations from a chimera compared with that in leukocytes from a nontransplanted mouse. (B) The degree of CSF inflammation at 24 hours after meningitis induction in $\mathrm{BM}$ chimeric mice is determined by the genotype of the BM donor. Note that the levels of chimerism were comparable among all 4 groups of chimeras. CSF pleocytosis correlated negatively with TRAIL deficiency in hematopoietic cells. (C) Real-time PCR revealed presence of TRAIL mRNA in wild-type and TRAIL-/- mice transplanted with wild-type BM (C57/C57 and TRAIL-1-/C57, respectively) but absence of TRAIL mRNA expression in TRAIL ${ }^{--}$mice transplanted with TRAIL ${ }^{--}$BM (TRAIL $\left.{ }^{-1-/ T^{2} A I L}{ }^{--}\right)$. bps, base pairs.

by pretreatment with a monoclonal anti-Ly6G antibody (Figure 4I) reduced apoptosis in the dentate gyrus at 24 hours after PCW administration compared with that in nondepleted TRAIL $^{-/}$mice treated with isotype control antibody. In the nondepleted mice, administration of $1 \mu \mathrm{g}$ rTRAIL significantly reduced the number of apoptotic cells in the dentate gyrus after experimental meningitis while no effect of rTRAIL was observed in granulocyte-depleted mice with meningitis. These findings confirm the important role of leukocytes in the induction of apoptosis in the hippocampus during nonreplicative bacterial meningitis. Moreover, they suggest that TRAIL is not a primary effector of granulocyte-induced cell death.

Effect of rTRAIL on the activation of murine granulocytes. In order to assess potential effects of TRAIL on the activation of granulocytes, we studied the regulation of CD18 surface antigen expression using FACS analysis of peripheral blood leukocytes from wild-type mice $(n=7)$. Granulocytes were identified by the coexpression of high levels of CD $11 \mathrm{~b}$ and Gr-1 antigens (CD $11 \mathrm{~b}^{\text {high }} / \mathrm{Gr}-1^{\text {high }}$ ). Within the $\mathrm{CD} 11 \mathrm{~b}^{\text {high }} / \mathrm{Gr}-1^{\text {high }}$ population, 2 subpopulations of granulocytes with low versus high expression of CD18 antigen were distinguishable in all samples (data not shown). The fraction of CD18 high granulocytes increased from $8.4 \% \pm 4.4 \%$ in unstimulated control samples to $22.6 \% \pm 1.5 \%$ after stimulation with $\mathrm{PCW}(P<0.05$ versus control) and to $26.6 \% \pm 0 \%$ after stimulation with $1 \mu \mathrm{M}$ PMA for 10 minutes $(P<0.05$ versus control). We did not observe any significant effects of rTRAIL in concentrations between 5 and $50 \mathrm{ng} / \mathrm{ml}$ on the proportion of CD18 $8^{\text {high }}$ granulocytes (data not shown). At higher doses, TRAIL induces granulocyte apoptosis (3).

Effect of TRAIL on in vitro granulocyte migration. We asked whether the presence of TRAIL modulates granulocyte motility and chemotaxis. In an in vitro transmigration assay, PCW-activated granulocytes from bone marrow (BM) aspirates migrated along a leukotriene $B_{4}$ gradient. The number of granulocytes present in the target compartment after 1 hour was not influenced by the TRAIL genotype of the cells or by preincubation with rTRAIL (data not shown).

Immunocytochemical analysis of TRAIL expression in meningitis. We aimed to identify the sources of TRAIL expression in experimental meningitis. Using immunocytochemistry, we observed TRAIL immunoreactivity on granulocytes and monocytes in the CSF of wild-type mice at 12 hours after meningitis induction (Supplemental Figure 3B). As expected, no TRAIL immunoreactivity was detected in TRAIL ${ }^{-/-}$mice with meningitis (data not shown). The majority of MPO-immunoreactive granulocytes in the tissue did not show staining by the anti-TRAIL antibody (data not shown).

Experiments in BM chimeric mice. Four groups of BM chimeric mice were generated by the transplantation of lethally irradiated wild-type or TRAIL ${ }^{-/-}$mice with BM harvested from wild-type or TRAIL ${ }^{-/-}$mice (donor to recipient: TRAIL $^{-/-}$to TRAIL $^{-/-}$; TRAIL $^{+/+}$ to $\mathrm{TRAIL}^{+/+}$; $\mathrm{TRAIL}^{-/-}$to $\mathrm{TRAIL}^{+/+}$; and $\mathrm{TRAIL}^{+/+}$to $\mathrm{TRAIL}^{-/-}$). Following in vitro transduction of hematopoietic cells with a murine stem cell virus-based retrovirus encoding the enhanced GFP, peripheral blood was analyzed for enhanced GFP expression 5 weeks after BM transplantation in order to determine the degree of chimerism (Figure 5A). Animals with less than $30 \%$ chimerism were excluded from the study. In the remaining animals, reconstitution of hematopoiesis with enhanced GFP-expressing peripheral blood cell progeny was on average $54 \% \pm 13 \%$ with no significant differences among the groups (Figure 5B). At 24 hours after meningitis induction, TRAIL $^{-/-}$mice reconstituted with TRAIL TH $^{-/}$BM cells had significantly more CSF pleocytosis than wild-type mice reconstituted with wild-type BM cells (Figure 5B). However, transplantation of TRAIL ${ }^{-/}$BM cells into wild-type mice (TRAIL ${ }^{-/-}$to TRAIL $^{+/+}$) increased CSF leukocyte counts at 24 hours to levels comparable to those of TRAIL ${ }^{-/-}$-to-TRAIL ${ }^{-/-}$mice and significantly higher than those of TRAIL TR $^{-/-}$-to-TRAIL ${ }^{-/}$mice. In contrast, transplantation of wild-type BM cells into TRAIL ${ }^{-/}$mice (TRAIL ${ }^{+/+}$to $\mathrm{TRAIL}^{-/}$) resulted in low CSF leukocyte counts that were not different from those of $\mathrm{TRAIL}^{+/+}$-to-TRAIL ${ }^{+/+}$mice but were significantly lower than those of TRAIL ${ }^{-/-}$-to-TRAIL ${ }^{-/-}$mice. Following extraction of total RNA from brain slices of BM chimeric mice at 24 hours after meningitis induction, RT-PCR analysis revealed the presence of TRAIL mRNA in $\mathrm{TRAIL}^{+/+}$-to-TRAIL ${ }^{+/+}$and in $\mathrm{TRAIL}^{+/+}$-to-TRAIL ${ }^{-/-}$mice but not in TRAIL Th- $^{-/-}$-TRAIL ${ }^{-/-}$mice (Figure $5 \mathrm{C}$ ). These experiments provide evidence that leukocytes are the primary source of TRAIL in meningitis and suggest an autocrine regulatory effect of leukocyte-derived TRAIL in acute CNS inflammation.

Findings in meningitis induced by live Streptococcus pneumoniae. Finally, we aimed to determine whether regulatory effects of the TRAIL system were also present in meningitis induced by live bacteria. In wild-type mice infected with the D39 strain of S. pneumoniae, bacterial concentrations in the CSF after 24 hours were not influ- 
A
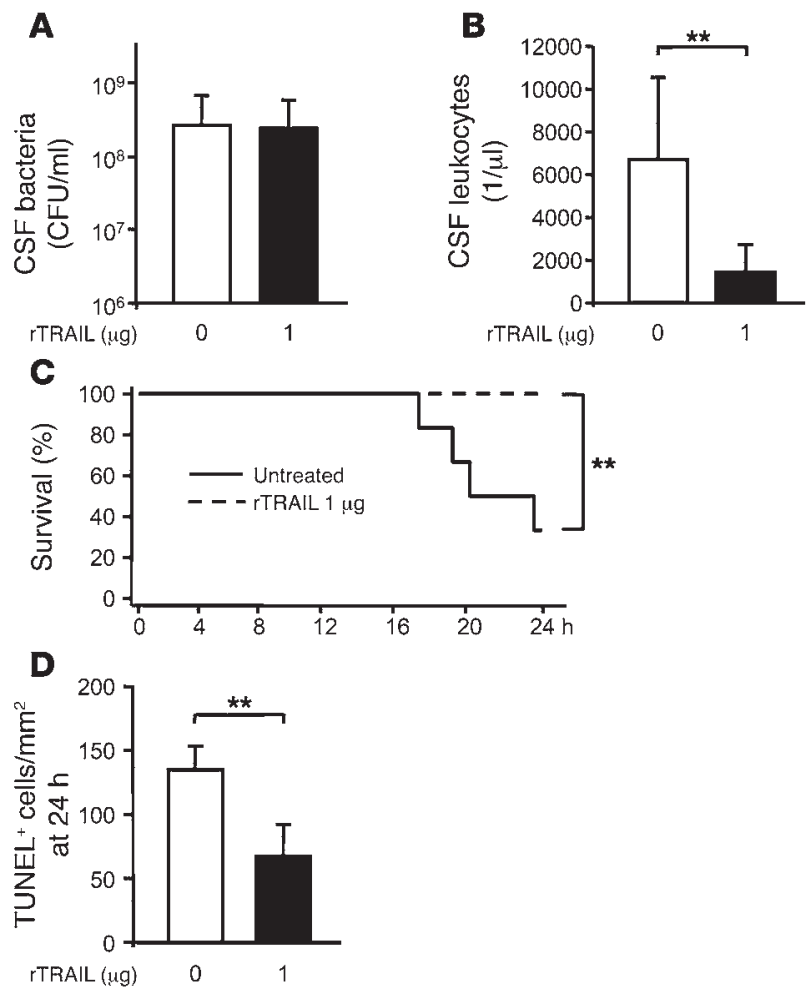

enced by intrathecal administration of $1 \mu \mathrm{g}$ rTRAIL at the time of meningitis induction (Figure 6A). Leukocyte concentrations in the CSF at the end of the experiment (Figure 6B) and mortality of meningitis (Figure 6C) were significantly lower in rTRAIL-treated mice than in untreated mice with meningitis. Moreover, treatment with rTRAIL reduced the number of TUNEL-positive cells in the dentate gyrus at 24 hours after infection (Figure 6D).

\section{Discussion}

Deficiency in TRAIL protracted acute inflammation and increased apoptosis in the hippocampus in experimental meningitis. These deleterious changes were reversed by rTRAIL or by the transplantation of TRAIL-expressing BM cells into a chimeric mouse model. Administration of rTRAIL into the subarachnoid space of wildtype mice with meningitis also reduced inflammation and apoptosis. We therefore identify TRAIL as a novel antiinflammatory cytokine in meningitis. Interestingly, increased concentrations of sTRAIL were found in patients with proven bacterial meningitis.

A major finding of our study is that TRAIL modifies the inflammatory response during experimental meningitis by regulating the recruitment and the survival of leukocytes. Previous ex vivo studies have also provided evidence for a role of TRAIL in the regulation of leukocyte survival. Human neutrophils express the death domain-containing receptor, TRAIL-R2 and membrane-bound TRAIL, which is released in soluble form by unknown proteases (14). We confirmed the presence of TRAIL-R2 on murine granulocytes. TRAIL and TRAIL death receptors promote apoptotic death of senescent circulating neutrophils upon their return to the BM (13). Activated tissue neutrophils have an extended life span due to the action of external survival signals such as GM-CSF, complement C5a, LPS, and type 1 interferons $(36,37)$ as well as temporary resistance to CD95 signaling (38). In vitro, initiation of apoptosis was

\section{Figure 6}

Effects of treatment with rTRAIL in meningitis induced by live pneumococci. (A) At 24 hours after infection, bacterial load in the CSF in untreated and rTRAIL-treated wild-type mice did not differ. (B) CSF leukocyte concentration was significantly lower in rTRAIL-treated mice than in controls at 24 hours after infection with live pneumococci. ${ }^{\star \star} P<0.01$, Student's $t$ test. (C) During the experimental period of 24 hours, mortality was higher in untreated versus rTRAIL-treated mice with meningitis. ${ }^{* \star} P<0.01$, log rank test. (D) Apoptosis in the dentate gyrus at 24 hours after meningitis induction was reduced by treatment with $1 \mu \mathrm{g}$ rTRAIL. ${ }^{* *} P<0.01$, Student's $t$ test.

reported to be regulated by TNF- $\alpha$ through increased shedding of membrane-bound TRAIL and gradual downregulation of the decoy receptor TRAIL-R3 thereby promoting death signaling via TRAILR2 (14). This is consistent with our findings of prolonged survival of activated CSF leukocytes in TRAIL-/- mice during meningitis and the increase of sTRAIL in the CSF of patients suffering from meningitis. A further observation of our study was the delayed onset of meningeal inflammation in $\mathrm{TRAIL}^{-/}$mice. The recruitment of neutrophils - otherwise the first line of defense in bacterial meningitis - was affected most prominently by the absence of TRAIL. There are conflicting reports with regard to the modulatory effects of TRAIL on the activation of endothelial cells during inflammation and the subsequent immigration of leukocytes. One study reported induction of apoptosis and NF-KB-dependent inflammatory gene expression by TRAIL (39). A further study described markedly decreased production of the chemokines CCL8/monocyte chemoattractant protein-2 (MCP-2) and CXCL10/IP-10, leading to a loss of the proadhesive activity of TNF- $\alpha$ in the presence of TRAIL (40). TRAIL could also exert a regulatory function on leukocyte activation, e.g., expression of adhesion molecules, motility, or chemotaxis. Inhibitory effects of TRAIL on the activation of autoantigen-specific $\mathrm{T}$ cells have recently been described (8). However, we did not detect a direct effect of rTRAIL on CD18 expression on peripheral blood granulocytes. CD18 is considered to be a marker of activation and adhesiveness. Also, neither the TRAIL genotype nor the addition of rTRAIL had an influence on granulocyte motility along a leukotriene $\mathrm{B}_{4}$ gradient in cell migration experiments. Thus, the exact mechanisms of action leading to different leukocyte kinetics in the presence and absence of TRAIL remain unclear at this time. However, based on the results obtained with BM chimeric mice, we conclude that the regulatory effect of TRAIL on the duration of the inflammatory process is mediated primarily within the leukocyte population. Leukocytes are both the relevant source and target of TRAIL since the inflammatory phenotype in the CSF and the detection of TRAIL mRNA in brains with meningitis were influenced by the genotype of the donor but not of the recipient mice. In patients with meningitis, the concentration of sTRAIL correlated with the number of leukocytes in the CSF. A contribution of BM-derived microglia as a source of TRAIL in the brains of chimeric mice appears unlikely as TRAIL is not normally found in brain tissue (41), and due to the rather short interval between BM transplantation and the induction of meningitis, only a very small number of these cells could be expected to derive from the transplanted hematopoietic cells (42).

Another important finding of our study is that TRAIL not only modifies the inflammatory response, but also determines the degree of brain damage in experimental meningitis. In the case of S. pneumoniae, the most frequent and invasive pathogen in bacte- 
rial meningitis, neuronal injury is driven by bacterial toxins and by the inflammatory host response $(25-27,43)$. On the host side, release of free radicals, proteases, and neurotoxic cytokines by immune cells contributes to the damage $(32,44)$. TNF- $\alpha$ caused hippocampal neurodegeneration in an infant rat model of meningitis $(45,46)$, but no conclusive data are available on the role of CD95 and TRAIL systems. TRAIL receptors but not TRAIL are expressed in the healthy brain (41). The addition of high concentrations of TRAIL to human living brain slices increases neuronal apoptosis (47). Moreover, TRAIL has been identified as a mediator of neuronal cell death in experimental autoimmune encephalomyelitis (48). Thus, TRAIL released by immune-competent cells may confer death signals to neurons during inflammation. However, our findings of increased apoptosis in the hippocampus of TRAIL $^{-/-}$mice with meningitis and of reduced apoptosis in wildtype mice treated with rTRAIL during meningitis argue against a prominent direct contribution of TRAIL signaling to neuronal cell death during acute meningitis. On the contrary, TRAIL may limit host-induced cytotoxicity by supporting the resolution of inflammation through enhanced apoptotic clearance of leukocytes. The importance of leukocytes as effectors of host-driven neurotoxicity is supported by our observation of reduced apoptosis in the hippocampus following blockade of leukocyte recruitment with a monoclonal antibody against CD18 or following granulocyte depletion in nonreplicative PCW-induced meningitis. These findings are in line with previously published data in other models of meningitis $(15-17,25,46,49)$. However, our histological findings suggest an indirect role of invading leukocytes, since MPO-positive granulocytes were not found in the vicinity of apoptotic cells in the brain in untreated meningitis. Moreover, the reduction of meningitisinduced apoptosis in the dentate gyrus of granulocyte-depleted mice was clearly independent of TRAIL. An additional protective mechanism of TRAIL besides enhanced leukocyte clearance may be the modulation of local inflammation within the brain parenchyma, as suggested by the elevated levels of cytokine gene expression in TRAIL ${ }^{-/-}$mice with meningitis. Along these lines, we and others (50) detected expression of TRAIL-R2 on CNS microglia.

In conclusion, we describe a new role for TRAIL as a modulator of granulocyte-driven inflammation. TRAIL limits the life span of activated leukocytes by its proapoptotic abilities. Furthermore, TRAIL appears to enhance granulocyte recruitment by as yet unknown mechanisms. The TRAIL pathway may therefore carry significant therapeutic potential for terminating an acute inflammatory response and improve the outcome of invasive infections.

\section{Methods}

Measurement of TRAIL in human CSF. Stored supernatants $\left(-80^{\circ} \mathrm{C}\right)$ of CSF from 11 patients with acute bacterial meningitis were included in the analysis. Patients were recruited for study according to their diagnosis at discharge and had to meet the following criteria: (a) clinical syndrome consistent with bacterial meningitis; and (b) presence of more than 1000 leukocytes/ $\mu \mathrm{l}$ or cultural identification of the pathogen (S. pneumoniae, $n=5$; Enterococcus faecalis, $n=1$; Neisseria meningitidis, $n=2$; unknown, $n=3$ ). For controls, we included 11 patients in whom CSF analysis had revealed normal results (35). Patients had given informed consent to the lumbar puncture, and use of the samples in studies was approved by Ethikkommission der Charité - Universitätsmedizin Berlin. TRAIL concentrations in the CSF were measured in duplicate in a blinded fashion, using a specific ELISA for TRAIL according to the manufacturer's protocol (R\&D Systems). A control serum sample was applied consistently to exclude plate-to-plate variations.
Bacterial culture and preparation of PCWs. In experiments involving live bacteria, an encapsulated strain of $S$. pneumoniae serotype 2 (strain D39, originally derived from a clinical isolate) was used, while PCWs were prepared from nonencapsulated pneumococci (strain R6). Bacteria were grown overnight in standard casein plus yeast medium at $37^{\circ} \mathrm{C}$ with $5 \%$ $\mathrm{CO}_{2}$. To prepare viable inocula, bacteria were pelleted during log phase growth and resuspended in pyrogen-free PBS. CFUs/ $\mu 1$ were determined photometrically (optical extinction at $620 \mathrm{~nm}$ ) using a standard curve, and serial dilutions were used to prepare defined inocula of $10^{6} \mathrm{CFUs} /$ animal in volumes of $40 \mu \mathrm{l}$. Correctness of the CFU calculations was verified by plating of serial dilutions.

Purified PCW was prepared as described $(15,51)$. Following heat inactivation, bacteria were mechanically disintegrated. The suspension was digested with $10 \mu \mathrm{g} / \mathrm{ml}$ DNase (Promega) and $50 \mu \mathrm{g} / \mathrm{ml} \mathrm{RNase} \mathrm{(USB} \mathrm{Corp.)} \mathrm{for}$ 1 hour at $37^{\circ} \mathrm{C}$ followed by treatment with $0.01 \%$ trypsin (Sigma-Aldrich) for 2 hours at $37^{\circ} \mathrm{C}$. The digest was sedimented by centrifugation $(23,000 \mathrm{~g}$, $20 \mathrm{~min}$ ) and resuspended in $2 \% \mathrm{SDS}$ (SERVA) at $90^{\circ} \mathrm{C}$ for 20 minutes followed by 8 cycles of washing. Using a standard curve, the optical density of the solution was adjusted to correspond to a concentration of $10^{7} \mathrm{CFUs} / 40$ $\mu \mathrm{l}$. The chemical composition of this cell wall has been published (52).

Animal experiments. Studies were conducted in a well-characterized model of nonreplicative pneumococcal meningitis (35). Animal care, use for experiments, and all experimental protocols were reviewed and approved by Landesamt für Gesundheit und Soziales, Berlin, Germany. Meningitis was induced in 8- to 12-week-old male C57BL/6 mice or TRAIL-- mice (backcrossed for more than 8 generations to the C57BL/ 6 background; ref. 45) by instillation of $40 \mu \mathrm{l} \mathrm{PCW}$ preparation equivalent to $10^{7} \mathrm{CFU}$ s into the lumbar spinal canal as described (35). Controls received an equal volume of pyrogen-free PBS. Adequate awakening from the anesthesia and absence of pareses were verified clinically. A subset of animals was treated with rTRAIL (45), which was injected intrathecally at the time of meningitis induction. To minimize potential contamination with LPS, a commercially available LPS removal kit (End-X Endotoxin Affinity Resin; Associates of Cape Cod) was used according to the manufacturer's instructions. In some animals, leukocyte recruitment was blocked by injection of a monoclonal antibody directed against CD18 (clone GAME46; BD Biosciences - Pharmingen). The antibody was injected into the tail vein at a concentration of $1 \mathrm{mg} / \mathrm{kg}$ at the time of meningitis induction, followed by repeated intraperitoneal injections of $0.1 \mathrm{mg} / \mathrm{kg}$ antibody after 6,12 , and 18 hours. Additional experiments were conducted in granulocyte-depleted mice. For this purpose, TRAIL $\mathrm{T}^{-/}$mice $(n=10)$ received a single intraperitoneal injection of a monoclonal rat antiLy6G antibody (BD Biosciences - Pharmingen; $0.1 \mathrm{mg} /$ animal) at 24 hours prior to surgery. Efficiency of this procedure was verified by hematological examination of peripheral blood for the presence of less than $1 \%$ granulocytes at the time of meningitis induction. TRAIL $-/$ mice $(n=10)$ injected with nonspecific rat IgG (BD Biosciences - Pharmingen; $0.1 \mathrm{mg} /$ animal) were used as nondepleted controls.

At the end of the experiments, animals were examined using the tightrope test (53). In this global motor and coordination task, the length of time during which a mouse is able to hold on to a horizontally suspended length of string is measured. Mice are lifted by the tail and released once they have firmly gripped the rope with both forepaws. A padded box is used to catch falling mice. The experiment was terminated after 30 seconds. Following this test, the animals were again deeply anesthetized. After dissection of the occipital muscles under a preparation microscope, a CSF sample was drawn from the cisterna magna using a $28 \mathrm{G}$ butterfly cannula attached to a microliter syringe. Obtained CSF samples ranged between 10 and $20 \mu \mathrm{l}$ /mouse. Leukocytes in the CSF were counted using a Fuchs-Rosenthal chamber at 400-fold magnification. Following transcardial perfusion with cold PBS, brains were harvested and snap frozen using methylbutane and dry ice. 
Generation of BMchimeric mice. Chimeras were generated as described $(48,54)$. In brief, BM cells from 8- to 12-week-old male donor mice pretreated

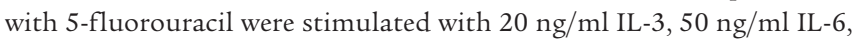
and $50 \mathrm{ng} / \mathrm{ml} \mathrm{SCF}$ (PromoCell) for 48 hours. In order to facilitate the assessment of chimerism, donor BM cells were genetically modified with a murine stem cell virus-based (MSCV-based) retroviral vector encoding the enhanced GFP. BM cells were cocultured with irradiated (1,300 cGy) $\mathrm{GP}+\mathrm{E} 86$ viral producer cells. After another 48 hours, BM cells were recovered from the cultures, and $5 \times 10^{6}$ nonadherent BM cells were transplanted by tail vein injection into lethally irradiated ( 2 doses of $550 \mathrm{cGy}$ separated by 3 hours) recipient mice. Five weeks after BM transplantation, peripheral blood was examined for enhanced GFP expression by FACS (FACSCalibur; BD). Dead cells (propidium iodide positive) were excluded. Data were evaluated using CellQuest software (version 3.1; BD). Only animals with more than $30 \%$ of chimerism were used for experiments.

CSF studies. Viability of CSF leukocytes was assessed by AOEB staining. $\mathrm{AO}$ and $\mathrm{EB}$ are fluorescent intercalating DNA dyes. While AO stains all nuclei green, EB is excluded by cells with an intact cell membrane. Double staining with $\mathrm{EB}$ and $\mathrm{AO}$ allows for differentiation of live, apoptotic, and necrotic cells (26): live cells have green, regular-sized nuclei. Nuclear condensation or fragmentation identifies apoptotic cells, and red staining identifies late apoptotic or necrotic cells. For AOEB staining, CSF samples were diluted 1:20 in 0.1\% PBS in 96-well cell culture plates. $\mathrm{AO}$ and EB were added to a final concentration of $2 \mu \mathrm{g} / \mathrm{ml}$ each. In additional samples, TUNEL reaction was used to label DNA strand breaks, a hallmark of apoptosis. For this purpose, CSF samples were air dried and briefly fixed with $4 \%$ paraformaldehyde on coated glass slides. TUNEL reaction was performed using a commercially available kit (Qbiogene) according to the manufacturer's instructions. A FITC conjugate was used for visualization. Hoechst $33258(1: 10,000)$ was employed as a nuclear counterstain. To determine whether TRAIL abbreviates the lifespan of activated CSF leukocytes, CSF was obtained from TRAIL $^{-/}$mice 12 hours after intrathecal challenge. Due to the small volumes, samples were pooled and diluted 1:10 in RPMI medium. rTRAIL $(100 \mathrm{ng} / \mathrm{ml})$ was added to one half of the sample (4). Following incubation at $37^{\circ} \mathrm{C}$ for $0,2.5,5$, and 8.5 hours, the percentage of dead cells was determined in the TRAIL-exposed and untreated sample using AOEB staining as above.

For immunocytochemical detection of TRAIL on CSF leukocytes, cells were fixed on to glass slides and incubated with an antibody against TRAIL (clone N2B2; eBioscience) at a dilution of 1:100 for 1 hour at ambient temperature. A Texas red-conjugated anti-rat secondary antibody was used for visualization, and Hoechst 33258 was used as a nuclear counterstain. To control for nonspecific binding of the conjugate, additional slides were processed with omission of the primary antibody. CSF leukocytes from TRAIL $/-$ mice were used as a negative control.

Histology and immunohistochemistry. To determine the extent of apoptosis in the dentate gyrus, $20-\mu \mathrm{m}$ cryosections were thaw-mounted on coated glass slides. Following fixation in $-20^{\circ} \mathrm{C}$ methanol for 10 minutes, slides were processed for TUNEL reaction using a commercially available fluorescent kit (Qbiogene). Slides were counterstained with Hoechst 33258 $(1: 10,000)$. TUNEL-positive condensed or fragmented nuclei were counted in the dentate gyrus and hilus region of both hemispheres on multiple sections per animal in a blinded fashion. The area of the dentate gyrus was determined using Stereo Investigator software (version 5.05.4; MBF Bioscience). Apoptotic cells were then divided by the dentate gyrus area to allow interindividual comparisons. For immunohistochemistry, sections were treated with $3 \%$ normal goat serum and $0.3 \%$ Triton X-100 (SigmaAldrich) at room temperature for 1 hour. Granulocytes were stained using a rabbit antibody against MPO (Chemicon) at a dilution of $1: 300$ at $4{ }^{\circ} \mathrm{C}$ overnight. A monoclonal hamster anti-mouse antibody against TRAIL-R2 (clone MD5-1; eBioscience) was used at a concentration of 1:100. Texas red-conjugated goat anti-hamster antibody (Vector Laboratories) and Alexa Fluor 488-conjugated goat anti-rabbit antibody (Invitrogen) were added at a concentration of 1:100 at room temperature for 1 hour to allow visualization of the staining. For double stainings, tissue was first processed for MPO immunohistochemistry using a Texas red conjugate followed by the TUNEL technique as described above.

RNA isolation and real-time PCR. Total RNA was extracted from cerebellar lysates using the TriFast method (peqGold TriFast; PEQLAB) following the manufacturer's instructions. First-strand cDNA was synthesized in a volume of $20 \mu \mathrm{l}$ using $1 \mu \mathrm{g}$ of total RNA and TaqMan reverse transcription reagents (Applied Biosystems). The RNA was quantified at $260 \mathrm{~nm}$ in a NanoDrop spectrophotometer at an OD 260/280 ratio of 1.7 to 2.0 for all samples. PCR was performed with an ABI PRISM 7000 (Applied Biosystems) and $2 \times$ qPCR MasterMix (Eurogentec) according to the manufacturers' instructions. To quantify target mRNA levels, TGF- $\beta$, TNF- $\alpha$, IFN- $\gamma$, IL-10, and IL-4 TaqMan Gene Expression assays were purchased from Applied Biosystems. The sequences CXCL12 (forward) 5'-CCAGAGCCAACGTCAAGCA-3', CXCL12 (reverse) 5'-TGCACACTTGTCTGTTGTTGTTCTT-3', and CXCL12 (probe) 5'-Fam-CTCAACACTCCAAACTGTGCCCTTCAGA-TAMRA-3' were designed with Primer Express software (version 2.0; Applied Biosystems). All primers spanned an intron to ensure discrimination between cDNA and genomic DNA. The relative amount of specific mRNA was normalized to GAPDH using the following sequences: GAPDH (forward) 5'-CTCAACTACATGGTCTACATGTTCCA-3'; GAPDH (reverse) 5'-CCATTCTCGGCCTTGACTGT-3'; and GAPDH (probe) 5'Fam-TGACTCCACTCACGGCAAATTCAACGT-TAMRA-3'. All PCR reactions were run in duplicate and were performed with 40 cycles, including a negative control consisting of PCR-grade water. Quantitative real-time PCR analysis was carried out using the 2- ${ }^{\Delta \Delta \mathrm{Ct}}$ method (55).

In vitro studies of murine blood. Differential hemograms were obtained microscopically in a Neubauer counting chamber after diluting peripheral blood samples in Turk's solution (1:20). In order to differentiate leukocyte subpopulations in the CSF, samples were air dried on untreated glass slides and stained with May-Grünwald and Giemsa solutions. For each animal, 200 leukocytes were assessed at 400-fold magnification.

Peripheral blood of naive C57BL/6 mice $(n=7)$ was collected in PBS with 2 mM EDTA. For the detection of TRAIL-R2 on neutrophils, samples were subjected to erythrocyte lysis and incubated with anti-CD14/CD32 antibody (BD Biosciences - Pharmingen) at a concentration of 1:50 to prevent nonspecific binding. Granulocytes were identified by their high Gr-1 expression using a PE-labeled anti-Gr-1 monoclonal antibody (BD Biosciences Pharmingen) at a dilution of $1: 100$ at $4^{\circ} \mathrm{C}$ for 25 minutes. The expression of TRAIL-R2 was analyzed by flow cytometry using a polyclonal goat antibody against TRAIL-R2 (ALEXIS Biochemicals) at a dilution of 1:200 at $4{ }^{\circ} \mathrm{C}$ for 30 minutes. Leukocytes not incubated with one or both primary antibodies served as controls. Samples were analyzed by 4-color flow cytometry on a FACSCalibur, and 8,000 gated granulocytes were measured.

To determine the effect of TRAIL on granulocyte activation, murine peripheral blood leukocytes were harvested as above and transferred to RPMI 1640 medium (Invitrogen). For stimulation, cells were incubated at $37^{\circ} \mathrm{C}$ for 10 minutes with PCW (prepared as above, 1:20 dilution), rTRAIL, or both. As a positive control, $1 \mu \mathrm{M}$ PMA (Sigma-Aldrich) was used for maximum stimulation. After washing the cells, samples were incubated for 30 minutes with APC-labeled anti-Gr-1, PE-labeled anti-CD11b, and FITC-labeled anti-CD18 antibodies (BD Biosciences - Pharmingen) at a dilution of 1:20. After washing, cells were analyzed on a FACSCalibur. $\mathrm{CD} 11 \mathrm{~b}^{\text {high }} / \mathrm{Gr}-1^{\text {high }}$ granulocytes were gated and analyzed for the expression of CD18 as a marker of activation. CellQuest software (version 3.1; $\mathrm{BD})$ was used for quantification. 
To study the effect of TRAIL on migratory capacity, BM cells were obtained from femurs and tibiae of wild-type and TRAIL ${ }^{-/-}$mice by flushing with RPMI supplemented with $10 \%$ FBS. Erythrocytes were depleted using ammonium chloride. BM cells were activated by incubation with PCW (1:20 dilution) for 12 hours at $37^{\circ} \mathrm{C}$ with or without addition of $1 \mu \mathrm{g} / \mathrm{ml}$ rTRAIL. We added $200 \mu \mathrm{l}$ of cell suspension, equivalent to 200,000 cells, to transwell polycarbonate inserts $(5-\mu \mathrm{m}$ pore size; Costar) and placed these inserts into 24-well plates containing $600 \mu \mathrm{l}$ /well of RPMI with $10 \%$ FBS and $100 \mathrm{nM}$ leukotriene B4 (Cayman Chemical). Cells were then allowed to migrate at $37^{\circ} \mathrm{C}$ for 1 hour. At the end of the experiment, granulocytes in the lower compartment were quantified using flow cytometry by study of the expression of the granulocyte marker Gr-1 as above.

Statistics. For 2-group comparisons, Student's $t$ test was used in the presence of normal distribution (Kolmogorov-Smirnov test); otherwise, MannWhitney $U$ test was used. ANOVA was consistently used for multigroup comparisons. To compare groups with equal variance, 1-way ANOVA was used followed by Student-Neuman-Keuls post hoc analysis. In samples failing equal variance testing, Kruskal-Wallis ANOVA on ranks was used followed by Dunn's post hoc analysis. Two-way ANOVA was used to test for an independent effect of rTRAIL on neuronal apoptosis in the granulocyte-depleted mice. Spearman's $r$ was used to describe the correlation between leukocyte count and sTRAIL concentration in patients. Multiple regression analysis was used to determine the relative impact of genotype and CSF leukocyte count on the number of apoptotic cells in the dentate gyrus. We performed log rank tests to compare survival between untreated and rTRAIL-treated mice with meningitis induced by live pneumococci and to test differences between groups in the motor coordination task (tightrope test).

\section{Acknowledgments}

This work was supported by grants from the German Research Council (SFB 507), the German Ministry of Science (BMBF), and the Meningitis Research Foundation. We thank Andrew Mason for reading the manuscript as a native English speaker.

Received for publication September 15, 2006, and accepted in revised form April 10, 2007.

Address correspondence to: Joerg R. Weber, Department of Cell Biology and Neurobiology, Center for Anatomy, Charité - Universitätsmedizin Berlin, Charitéplatz 1, 10117 Berlin, Germany. Phone: 49-30-450-528002; Fax: 49-30-450-528902; E-mail: joerg.weber@charite.de.

Olaf Hoffmann and Josef Priller contributed equally to this work.

Frauke Zipp and Joerg R. Weber contributed equally to this work.
1. Bouralexis, S., Findlay, D.M., and Evdokiou, A. 2005. Death to the bad guys: targeting cancer via Apo2L/TRAIL. Apoptosis. 10:35-51.

2. Wendling, U., et al. 2000. Expression of TRAIL receptors in human autoreactive and foreign antigen-specific T cells. Cell Death Differ. 7:637-644.

3. Hasegawa, H., et al. 2004. Restricted expression of tumor necrosis factor-related apoptosis-inducing ligand receptor 4 in human peripheral blood lymphocytes. Cell. Immunol. 231:1-7.

4. Renshaw, S.A., et al. 2003. Acceleration of human neutrophil apoptosis by TRAIL. J. Immunol. 170:1027-1033.

5. Lum, J.J., et al. 2001. Induction of cell death in human immunodeficiency virus-infected macrophages and resting memory CD $4 \mathrm{~T}$ cells by TRAIL/ Apo2l. J. Virol. 75:11128-11136.

6. Griffith, T.S., et al. 1999. Monocyte-mediated tumoricidal activity via the tumor necrosis factor-related cytokine, TRAIL. J. Exp. Med. 189:1343-1354.

7. Takeda, K., et al. 2002. Critical role for tumor necrosis factor-related apoptosis-inducing ligand in immune surveillance against tumor development. J. Exp. Med. 195:161-169.

8. Lünemann, J.D., et al. 2002. Death ligand TRAIL induces no apoptosis but inhibits activation of human (auto)antigen-specific T cells. J. Immunol. 168:4881-4888.

9. Hilliard, B., et al. 2001. Roles of TNF-related apoptosis-inducing ligand in experimental autoimmune encephalomyelitis. J. Immunol. 166:1314-1319.

10. Song, K., Chen, Y., and Goke, R. 2000. Tumor necrosis factor-related apoptosis-inducing ligand (TRAIL) is an inhibitor of autoimmune inflammation and cell cycle progression. J. Exp. Med. 191:1095-1104.

11. Lamhamedi-Cherradi, S.E., Zheng, S.J., Maguschak, K.A., Peschon, J., and Chen, Y.H. 2003. Defective thymocyte apoptosis and accelerated autoimmune diseases in TRAIL ${ }^{(-)}$mice. Nat. Immunol. 4:255-260.

12. Wandinger, K.P., et al. 2003. TNF-related apoptosis inducing ligand (TRAIL) as a potential response marker for IFN-beta therapy in multiple sclerosis. Lancet. 361:2036-2043.

13. Lum, J.J., Bren, G., McClure, R., and Badley, A.D. 2005. Elimination of senescent neutrophils by TNF-related apoptosis-inducing ligand. J. Immunol.
175:1232-1238.

14. Kamohara, H., et al. 2004. Regulation of tumour necrosis factor-related apoptosis-inducing ligand (TRAIL) and TRAIL receptor expression in human neutrophils. Immunology. 111:186-194.

15. Tuomanen, E.I., Saukkonen, K., Sande, S., Cioffe, C., and Wright, S.D. 1989. Reduction of inflammation, tissue damage, and mortality in bacterial meningitis in rabbits treated with monoclonal antibodies against adhesion-promoting receptors of leukocytes. J. Exp. Med. 170:959-969.

16. Saez-Llorens, X., et al. 1991. Enhanced attenuation of meningeal inflammation and brain edema by concomitant administration of anti-CD 18 monoclonal antibodies and dexamethasone in experimental Haemophilus meningitis. J. Clin. Invest. 88:2003-2011.

17. Weber, J.R., Angstwurm, K., Burger, W., Einhaupl, K.M., and Dirnagl, U. 1995. Anti ICAM-1 (CD 54) monoclonal antibody reduces inflammatory changes in experimental bacterial meningitis. J. Neuroimmunol. 63:63-68.

18. Bohr, V., et al. 1983. Sequelae from bacterial meningitis and their relation to the clinical condition during acute illness, based on 667 questionnaire returns. Part II of a three part series. J. Infect. 7:102-110.

19. De Gans, J., and van de Beek, D. 2002. Dexamethasone in adults with bacterial meningitis. N. Engl. J. Med. 347:1549-1556.

20. Scheld, W.M., et al. 1980. Cerebrospinal fluid outflow resistance in rabbits with experimental meningitis. Alterations with penicillin and methylprednisolone. J. Clin. Invest. 66:243-253.

21. Tauber, M.G., Khayam-Bashi, H., and Sande, M.A. 1985. Effects of ampicillin and corticosteroids on brain water content, cerebrospinal fluid pressure, and cerebrospinal fluid lactate levels in experimental pneumococcal meningitis. J. Infect. Dis. 151:528-534.

22. van de Beek, D., et al. 2002. Cognitive impairment in adults with good recovery after bacterial meningitis. J. Infect. Dis. 186:1047-1052.

23. Weisfelt, M., et al. 2006. Cognitive outcome in adults with moderate disability after pneumococcal meningitis. J. Infect. 52:433-439.

24. Nau, R., Soto, A., and Bruck, W. 1999. Apoptosis of neurons in the dentate gyrus in humans suffering from bacterial meningitis. J. Neuropathol. Exp. Neurol.
58:265-274.

25. Braun, J.S., et al. 1999. Neuroprotection by a caspase inhibitor in acute bacterial meningitis. Nat. Med. 5:298-302.

26. Braun, J.S., et al. 2001. Apoptosis-inducing factor mediates microglial and neuronal apoptosis caused by pneumococcus. J. Infect. Dis. 184:1300-1309.

27. Braun, J.S., et al. 2002. Pneumococcal pneumolysin and $\mathrm{H}_{2} \mathrm{O}_{2}$ mediate brain cell apoptosis during meningitis. J. Clin. Invest. 109:19-27. doi:10.1172/ JCI200212035.

28. van der Flier, M., Geelen, S.P., Kimpen, J.L., Hoepelman, I.M., and Tuomanen, E.I. 2003. Reprogramming the host response in bacterial meningitis: how best to improve outcome? Clin. Microbiol. Rev. 16:415-429.

29. Van de Beek, D., de Gans, J., McIntyre, P., and Prasad, K. 2007. Corticosteroids in acute bacterial meningitis. Cochrane Database Syst. Rev. 1:CD004405.

30. Viladrich, P.F., et al. 1991. Evaluation of vancomycin for therapy of adult pneumococcal meningitis. Antimicrob. Agents Chemother. 35:2467-2472.

31. Tunkel, A.R., and Scheld, W.M. 2002. Corticosteroids for everyone with meningitis? N. Engl. J. Med. 347:1613-1615

32. Nau, R., and Bruck, W. 2002. Neuronal injury in bacterial meningitis: mechanisms and implications for therapy. Trends Neurosci. 25:38-45.

33. Meagher, L.C., Cousin, J.M., Seckl, J.R., and Haslett, C. 1996. Opposing effects of glucocorticoids on the rate of apoptosis in neutrophilic and eosinophilic granulocytes. J. Immunol. 156:4422-4428.

34. Cox, G. 1995. Glucocorticoid treatment inhibits apoptosis in human neutrophils. Separation of survival and activation outcomes. J. Immunol. 154:4719-4725.

35. Hoffmann, O., et al. 2002. Triptans reduce the inflammatory response in bacterial meningitis. J. Cereb. Blood Flow Metab. 22:988-996.

36. Lee, A., Whyte, M.K., and Haslett, C. 1993. Inhibition of apoptosis and prolongation of neutrophil functional longevity by inflammatory mediators. J. Leukoc. Biol. 54:283-288.

37. Scheel-Toellner, D., et al. 2002. Cytokine-mediated inhibition of apoptosis in non-transformed $\mathrm{T}$ cells and neutrophils can be dissociated from protein 
kinase B activation. Eur. J. Immunol. 32:486-493.

38. Watson, R.W., Rotstein, O.D., Jimenez, M., Parodo, J., and Marshall, J.C. 1997. Augmented intracellular glutathione inhibits Fas-triggered apoptosis of activated human neutrophils. Blood. 89:4175-4181.

39. Li, J.H., Kirkiles-Smith, N.C., McNiff, J.M., and Pober, J.S. 2003. TRAIL induces apoptosis and inflammatory gene expression in human endothelial cells. J. Immunol. 171:1526-1533.

40. Secchiero, P., et al. 2005. TRAIL counteracts the proadhesive activity of inflammatory cytokines in endothelial cells by down-modulating CCL8 and CXCL10 chemokine expression and release. Blood. 105:3413-3419.

41. Dorr, J., et al. 2002. Lack of tumor necrosis factorrelated apoptosis-inducing ligand but presence of its receptors in the human brain. J. Neurosci. 22:RC209.

42. Priller, J., et al. 2001. Targeting gene-modified hematopoietic cells to the central nervous system: use of green fluorescent protein uncovers microglial engraftment. Nat. Med. 7:1356-1361.

43. Bermpohl, D., et al. 2005. Bacterial programmed cell death of cerebral endothelial cells involves dual death pathways. J. Clin. Invest. 115:1607-1615. doi:10.1172/JCI23223.

44. Quagliarello, V., and Scheld, W.M. 1992. Bacterial meningitis: pathogenesis, pathophysiology, and progress. N. Engl. J. Med. 327:864-872.

45. Bogdan, I., Leib, S.L., Bergeron, M., Chow, L., and Tauber, M.G. 1997. Tumor necrosis factor-alpha contributes to apoptosis in hippocampal neurons during experimental group B streptococcal meningitis. J. Infect. Dis. 176:693-697.

46. Leib, S.L., et al. 2001. Inhibition of matrix metalloproteinases and tumour necrosis factor alpha converting enzyme as adjuvant therapy in pneumococcal meningitis. Brain. 124:1734-1742.

47. Nitsch, R., et al. 2000. Human brain-cell death induced by tumour-necrosis-factor-related apoptosis-inducing ligand (TRAIL). Lancet. 356:827-828.

48. Aktas, O., et al. 2005. Neuronal damage in autoimmune neuroinflammation mediated by the death ligand TRAIL. Neuron. 46:421-432.

49. Zysk, G., et al. 1996. Anti-inflammatory treatment influences neuronal apoptotic cell death in the den- tate gyrus in experimental pneumococcal meningitis. J. Neuropathol. Exp. Neurol. 55:722-728.

50. Matysiak, M., Jurewicz, A., Jaskolski, D., and Selmaj, K. 2002. TRAIL induces death of human oligodendrocytes isolated from adult brain. Brain. 125:2469-2480.

51. Weber, J.R., et al. 2003. Recognition of pneumococcal peptidoglycan: an expanded, pivotal role for LPS binding protein. Immunity. 19:269-279.

52. Garcia-Bustos, J.F., Chait, B.T., and Tomasz, A. 1987. Structure of the peptide network of pneumococcal peptidoglycan. J. Biol. Chem. 262:15400-15405.

53. Miquel, J., and Blasco, M. 1978. A simple technique for evaluation of vitality loss in aging mice, by testing their muscular coordination and vigor. Exp. Gerontol. 13:389-396.

54. Priller, J., et al. 2001. Neogenesis of cerebellar Purkinje neurons from gene-marked bone marrow cells in vivo. J. Cell Biol. 155:733-738.

55. Livak, K.J., and Schmittgen, T.D. 2001. Analysis of relative gene expression data using real-time quantitative PCR and the 2(-Delta Delta C(T)) method. Methods. 25:402-408. 